1. FCPS II Trainee (Medicine)

2. FCPS (Medicine)

Associate Professor, LUMHS

3. M.D (Medicine)

4. $1^{\text {st }}$ year M.B.B.S Student

Department of Medicine

Liaquat University of Medical and

Health Sciences

Hyderabad/Jamshoro

Correspondence Address:

Dr. Ramesh Kumar Suthar

Flat no. 4, Yasir Palace,

Salawat Road,

Tando Wali Mohammad Hyderabad. rk22258627@gmail.com

Article received on: 26/10/2015

Accepted for publication:

03/12/2015

Received after proof reading:

13/01/2016

\section{CHRONIC HEPATITIS C; \\ "APRI SCORE A NONINVASIVE MARKER, ITS PREDICTIVE VALUE FOR DETERMINATION OF HEPATIC FIBROSIS AND LIVER CIRRHOSIS AT TERTIARY CARE HOSPITAL HYDERABAD/ JAMSHORO"}

\section{Dr. Muhammad Salim Rind', Dr. Muhammad Iqbal shah², Dr. Ramesh Kumar Suthar ${ }^{3}$, Syed Jahangir ${ }^{4}$}

ABSTRACT: The major causative factor of hepatic cirrhosis and its complications in chronic hepatitis $C$ is due to presence of liver fibrosis. To assess prognosis and management related decisions, the exact staging of liver fibrosis is of greatest importance. Now, liver biopsy is the inexact gold standard for this purpose. APRI or AST to platelet ratio Index is the best noninvasive marker which can predict presence of fibrosis in majority of chronic hepatitis $C$ patients without the need of biopsy. It is also cost effective and only depends on routine testing (Platelet count and serum AST). Objectives: To determine the positive predictive value of APRI Score for the prediction of fibrosis and cirrhosis in chronic hepatitis C patients. Study design: Cross sectional study. Setting: Medical wards and Out Patient Medicine Department of Liaquat University Hospital Hyderabad / Jamshoro. Period: 6 months. Methodology: A total of 51 patients of either gender, age $>14$ years presenting to Medical wards and OPD for the evaluation of chronic hepatitis were enrolled in this research study after giving preference and avoiding criteria. Clinical data was collected at the time of liver biopsy and blood samples for liver function tests, blood glucose and complete blood picture with platelet count were collected before the biopsy (only AST and platelet count are needed for APRI score) a core biopsy needle of 14 gauge was used and the procedure was conducted under ultrasound guidance. Fibrosis stage was determined according to the METAVIR group scoring system. The patients were examined in a very comfortable manner and all the information collected from the patients was kept confidential and entered in the predesigned proforma. Results: A total of 51 patients were selected for this study. Out of these $31(60.78 \%)$ were male and $20(39.22 \%)$ were female. The mean age was 42.53 years $( \pm 11.2 \mathrm{SD})$. The positive predictive value for APRI score between 0.5 to 1.0 was $58.82 \%$ whereas the positive predictive values for APRI score $1.1-1.5$ was $70.58 \%$. Conclusion: The positive predictive values of APRI score in the ranges of 0.5 to 1.5 were not indicative of the presence of significant liver fibrosis in this research study. However, additional data are required to authenticate or disprove the usefulness of APRI score for the prediction of significant hepatic fibrosis in chronic hepatitis $\mathrm{C}$ patients.

Key words: $\quad$ APRI score, liver biopsy, hepatic fibrosis, chronic hepatitis C

Article Citation: Rind MS, Shah MI, Suthar RK, Jahangir S. Chronic hepatitis C; "APRI score a noninvasive marker, its predictive value for determination of hepatic fibrosis and liver cirrhosis at tertiary care hospital Hyderabad/ Jamshoro. Professional Med J 2016;23(1):050-055. DOI: 10.17957/TPMJ/16.3139

\section{INTRODUCTION}

Throughout the world, high proportion of liver illnesses are due to hepatitis $\mathrm{C}$ virus which is the most grim infection. Approximately 170million persons of the world populace are infected with hepatitis $\mathrm{C}$ virus. ${ }^{1}$ In Pakistan more than 110 million persons suffer from Hepatitis C, founding $3.6 \%$ of the population. ${ }^{2}$ Genotype 3 is the most predominant in our population. ${ }^{3}$ Patients are at high risk (15-20\%) of developing chronic hepatitis, cirrhosis and hepatocellular carcinoma, if not properly treated in time. ${ }^{4}$ For initiation of treatment in patients of hepatitis $\mathrm{C}$, degree of liver fibrosis is the vital feature. ${ }^{5}$ Liver biopsy is the gold standard for recognizing the degree of fibrosis, but it is an invasive procedure and inter observer variation as well as sampling error and procedure related complications such as pain , bleeding and injury to near organs and certain contraindications limit its usefulness. ${ }^{6}$ The quest for a practical, noninvasive method that could be used as a substitute to liver biopsy has been the matter of various research studies. 
In 2003 Wai et al showed in research study in which they authenticated the index known as APRI Score that creates the relationship between serum aspartate aminotransferase levels and platelet count. The parameters are simple, cheap, inexpensive and easily available in the distant places. ${ }^{7}$ The AST to Platelet Ratio index (APRI) is calculated as (AST/upper limit of normal range)/platelet count $(10 \% / L) \times 100$. This index has been validated in the past as a substitute marker of significant liver fibrosis in HIV/HCV co infected patients, and has recently been used to determine advanced fibrosis in HIV co infected patient. ${ }^{8}$ Liver fibrosis is the most important agent for estimating clinical outcome in patients of hepatitis. The main objective of our this research study was to evaluate the diagnostic accuracy of APRI for hepatic fibrosis staging as compared to liver biopsy in the untreated Chronic Hepatitis C patients in tertiary care hospital at Hyderabad / Jamshoro.

\section{MATERIAL AND METHODS}

This cross sectional study was conducted in Medical wards of LUH Hyderabad/ Jamshoro. Duration of this research study was 6 months. Total of 51 patients after written consent and full explanation of research work were selected for this research study. Selected patients of both gender male and female and >14 years of age who were hepatitis $\mathrm{C}$ positive (via PCR) and until not received interferon and treatment for hepatitis $\mathrm{C}$, they were not alcoholics. Patients with hepatitis $B$ co infected with hepatitis $C$ (detected by HBsAg) and hepatocellular carcinoma (detected by ultrasonography of abdomen) and hepatic cirrhosis patients due to etiology other than Hepatitis $C$ were not included in this study.

The data with written consent were collected from the above said patients before liver biopsy and research study and blood sample for LFT, blood glucose, blood complete picture with platelet count taken and ultrasound of abdomen was also done.

The upper limit of normal (ULN) of AST was $38 \mathrm{U} / \mathrm{L}$ for men and 32U/L for women. Liver biopsy was performed under local anesthesia by a trained person, who had $>5$ years experience of performing such procedures. A core biopsy needle was of 14 gauge and procedure was conducted under U/S guidance. Adequate biopsy sample was defined as specimen size greater than $10 \mathrm{~mm}$ and more than 5 portal tracts. A single qualified histopathologist, who had more than 5 years experience of writing reports for such specimen and who was uninformed about the clinical data assessed by slides. Fibrosis stage was determined according to METAVIR group scoring system and would be classified as $\mathrm{FO}=$ no fibrosis, F1=portal fibrosis without septa, F2=few septa, F3=numerous septa without cirrhosis or F4=cirrhosis. The patients were examined in very comfortable manner and all the information collected from the patients was kept confidential and entered in predesigned proforma.

\section{DATA ANALYSIS}

Statistical package for social sciences (SPSS) version 16 was used for data processing purpose. Descriptive statistics included mean \pm standard deviation (SD) of continuous data i.e., age, blood sugar (RBS), AST level, platelet count. Frequencies will be calculated from the categorical data i.e., gender, grade of fibrosis, APRI would be divided into four categories $(<0.5$, $0.5-1,1-1.5$ and $>1.5$ ) and the effect modifiers like age, gender and diabetes mellitus were controlled through stratification.

\section{RESULTS}

This cross sectional study was conducted at Medical wards of Liaquat university hospital Hyderabad/Jamshoro. A total of 51 patients were selected for this research study. Out of $31(60.78 \%)$ were male and 20(39.22\%) were female. The mean age was 42.53 years $( \pm 11.2 S D)$. All patients were suffering from chronic hepatitis $C$ and were untreated. The patients were stratified according to age, sex and grade of liver fibrosis according to METAVIR scoring system. Table-I The different age groups were divided into age $<30$ years, between 31-50years and 51-70years respectively .The first group $(<30$ years) had a mean age of $27.44( \pm 1.3 S D)$ and had a total of 9 patients, 
second group(31-50 years) had a mean age of 40.24( \pm 5.2 SD) and had a 29 patients and last group(51-70) had a mean age of $58.08( \pm 5.05$ SD) and had a total of 13 patients. The patients were also divided on the basis of being diabetic or non-diabetic. Out of 51 , a total of $29(56.86 \%)$ had diabetes and whereas 22(43.14\%) were non diabetic. On the basis of APRI, the patients were divided into four groups, APRI <0.5(8patients) formed the first group, APRI 0.5-1.0(17 patients) second, APRI1.1-1.5 (also 17 patients) formed the third and >1.5 APRI, the fourth group (9 patients) respectively. The patients who were having $A P R I<0.5$ (the first group)and those who had APRI>1.5(the last group)were not subjected to the procedure of liver biopsy as the low APRI rules out hepatic fibrosis and high APRI confirms the presence of liver cirrhosis. So that a total of 34 patients were offered the procedure of liver biopsy and all agreed to go ahead. Out of these34 F0 grade was present in $7(20.59 \%)$ patients, whereas F1, F2, F3, F4 grades were present in $5(14.71 \%), 11(32.35 \%), 7(20.59 \%)$ and $4(11.76 \%)$ respectively. Significant fibrosis is taken as the presence of grade F2to F4. Table-II and III shows the calculation of positive predictive value for APRI category of 0.5-1.0 and 1.1-1.5 respectively. The value are 58.82 for APRI $0.5-1.0$ and 70.58 for APRI 1.1-1.5.

\section{DISCUSSION}

Liver fibrosis results from various types of hepatic injuries and a primary determinant of the outcome in chronic hepatic disease of advanced stage..$^{9-10}$

Due to vivid increase in the prevalence of chronic hepatitis $\mathrm{C}$, metabolic syndrome and Non alcoholic fatty liver disease, the process of liver biopsy has become unwieldly, overburdening and problematic because of the larger number of patients awaiting for liver biopsy procedure. Due to this extra stress is increased in the gastrointestinal units, operation theatres, Intensive Care Units and pathological laboratories. Because of this persistent search for noninvasive, simple, reliable biomarkers that can allow recognizing patients with liver fibrosis are greatly required. ${ }^{11}$

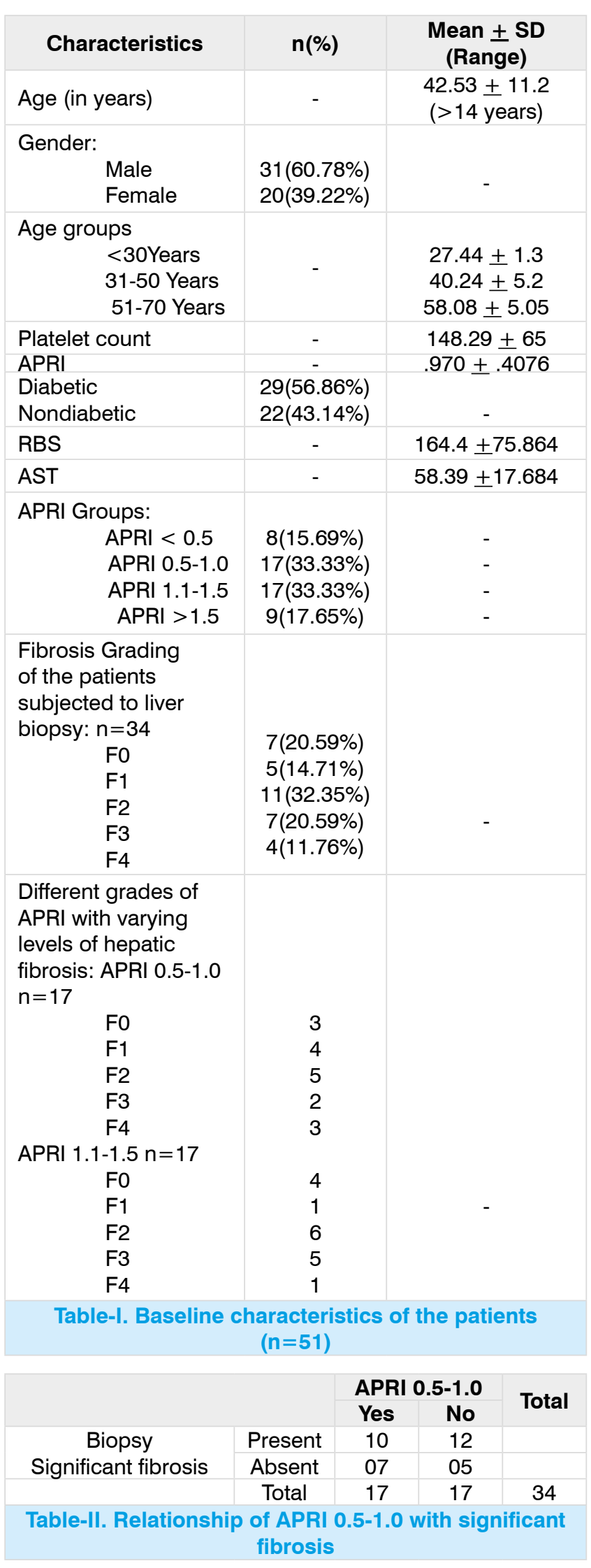


True Positive: biopsy show significant fibrosis +APRI 0.5-1.0

False Positive: biopsy negative for significant fibrosis +APRI 0.5-1.0

Positive predictive value: True Positive/False Positive + False Positive $\times 100$

$\mathrm{PPV}=58.82 \%$

\begin{tabular}{|c|c|c|c|c|}
\hline & & \multicolumn{2}{|c|}{ APRI 1.1-1.5 } & \multirow{2}{*}{ Total } \\
\cline { 2 - 5 } & & Yes & No & \\
\hline Biopsy & Present & 12 & 10 & \\
\hline Significant fibrosis & Absent & 05 & 07 & \\
\hline & Total & 17 & 17 & 34 \\
\hline
\end{tabular}

Table-III. Relationship of APRI 1.1-1.5 with significant fibrosis

True Positive: biopsy show significant fibrosis + APRI 1.1-1.5

False Positive: biopsy negative for significant fibrosis + APRI 1.1-1.5

Positive predictive value: True Positive/False

Positive + False Positive $\times 100$

$\mathrm{PPV}=70.58 \%$

The availability of such biomarkers has marvelous potential to completely change the diagnostic and monitoring approaches through the decreased requirement for liver biopsy. ${ }^{11}$

APRI score is tested for the prediction of hepatic fibrosis/ cirrhosis in a various clinical states. The APRI score relies on routine laboratory tests and therefore easily available in routine clinical practice. This index has been shown to predict the presence of significant fibrosis and cirrhosis in several investigations. ${ }^{12,13}$.These include chronic hepatitis $\mathrm{C}$, to assess the early viral response in hepatitis $C$, chronic hepatitis $C$ and HIV confection, chronic hepatitis $B$, nonalcoholic fatty liver disease, nonalcoholic steatohepatitis, hepatocellular carcinoma. Because of this reason, this versatile yet reasonable noninvasive biomarker for prediction of hepatic fibrosis was selected for this study. It is well recognized fact that this biomarker is tremendously effective in ruling out significant hepatic fibrosis (with values $<0.5$ ) and confirming the presence of cirrhosis (with values in excess of 1.5). ${ }^{14}$
It is relatively easy to rule out or predict fibrosis when extreme values of APRI are observed in chronic hepatitis $C$, however a gray area is present when the values lie around 0.5 to 1.5 . Specially because of this reason this research study was undertaken to try and clarify this unclear zone of APRI.

The positive predictive values for the value of 0.5 to 1.0 was $58.82 \%$, whereas for value of 1.1 to 1.5 it was $70.58 \%$. The former value was a disappointment as it missed $41.12 \%$ of patients who had significant fibrosis on biopsy, however the latter value $(70.58 \%)$ missed only29.42\%of patients who had significant hepatic fibrosis. In both cases significant numbers of patients are going to be missed if fibrosis is to be noted by APRI score alone. This result was in accordance with Sebastiani et $\mathrm{al}^{15}$ and Shaikh $\mathrm{S}$ et $\mathrm{al}^{16}$ respectively.

Mostly the various results and conclusions remained positive regarding the potential role of APRI in the forecast of fibrosis in chronic hepatitis $C$, however several research studies were conducted in western Europe or in the United States.

Therefore, it is doubtful and questionable whether the APRI score may be used in the noninvasive assessment of fibrosis within a wide range of chronic liver diseases throughout world. Shaikh et al have recently worked on several indirect biomarkers of liver fibrosis and APRI score was observed as very valuable and effective in the forecast of liver fibrosis. ${ }^{16}$

Wai et $\mathrm{al}^{17}$ resulted and corroborated the APRI score in a cohort study of patients with chronic hepatitis $\mathrm{C}$. In their research study the APRI score had a PPV of $80-88 \%$ for the diagnosis of significant hepatic fibrosis. Based on the high predictive values, the authors concluded that the APRI score can avoid liver biopsy in approximately $50 \%$ of the patients. ${ }^{17}$

Subsequently numerous researchers have tried their best to validate these findings but the results 
were contradictory. ${ }^{18}$

The differences in patients population including the prevalence of significant fibrosis and in the reference ranges for AST may in partly explain these inconsistencies.

These results are in good agreement with our data and advise that APRI score may be a beneficial noninvasive marker of liver fibrosis in this group of patient.

\section{CONCLUSION}

This study concludes that the positive predictive values of APRI in the ranges of 0.5 to 1.5 were not indicative of the significant liver fibrosis in this study. However further additional data are required to validate or disprove the valuable and usefulness of APRI score for the prediction of significant liver fibrosis in chronic hepatitis C patients.

\section{Copyright(C) 03 Dec, 2015.}

\section{REFERENCES}

1. Report of a WHO consultation organization in collaboration with the viral hepatitis prevention board, Antwerp, Belgium. Global surveillance and control of hepatitis C J Viral Hepat1999;6:35-47.

2. Waheed Y, Shafi T, Safi SZ, Qadri I. Hepatitis C virus in Pakistan: A systematic review of prevalence, genotypes and risk factors. World J Gastroenterol 2009; 15: 5647-53.

3. Idrees $M$, Riazuddin S. Frequency distribution of hepatitis $C$ virus genotypes in different geographical regions of Pakistan and their possible routes of transmission. BMC Infectious diseases 2008;8:69.

4. Chen, SL, Morgan, TR. The natural history of hepatitis C virus (HCV) infection. Int J Med Sci 2006;3(2):47-52.

5. Booth JC, Grady, O'J. and Neuberger J. Clinical guidelines on the management of hepatitis C. Gut 2001;49:1-21.

6. Bedossa P, Darge `re D, Paradis V. Sampling variability of liver fibrosis in chronic hepatitis C. Hepatology 2003; 38: 1449-57.

7. Wai CT, Greenson JL, Fontana RJ, Kalbfleich JD, Marrero JA, Conjeevaram HS, Lok AS. A simple noninvasive index can predict both significant fibrosis and cirrhosis in patients with chronic hepatitis C.
Hepatology 2003; 38: 518-26.

8. DallaPiazza M, Amorosa VK, Kostman JR,Lo Re V. Prevalence and risk factors for significant liver fibrosis among HIV-monoinfected patients. BMC Infect Dis 2010;10:116.

9. Iredale J. Defining therapeutic targets for liver fibrosis:exploiting the biology of inflammation and repair. Pharmacol Res.2008;58(2):129-36.

10. Castera L. Assessing liver fibrosis. Expert Rev Gastroenterol Hepatol.2008;2(4):541-52.

11. Parise ER, Oliveira AC, Figueiredo-Mendes C ,Lanzoni V, Martins $\mathrm{J}$, Nader $\mathrm{H}$,et al. Noninvasive serum markers in the diagnosis of structural liver damage in chronic hepatitis c virus infection. Liver Int.2006;26(9):1095-9.

12. Borsoi Viana MS,Takei K, Collarile Yamaguti DC, Guz $B$, Strauss $E$. Use of AST platelet ratio index(APRI score)as an alternative for liver biopsy for treatment indication in chronic hepatitis c. Ann Hepatol.2009;8(1):26-31.

13. Shaheen AA, Myers RP. Systemic review and meta analysis of the diagnostic accuracy of fibrosis marker panels in patients with HIV/hepatitis c coinfection. HIV clin Trials.2008;9(1):43-51.

14. Guha IN, Parkes J, Roderick P,Chattopadhyay D, Cross $\mathrm{R}$, Harris $\mathrm{S}$ et al. Non invasive marker of fibrosis in non alcoholic fatty liver disease:validating the European Liver Fibrosis panel and exploring simple markers. Hepatology 2008;47(2):455-460.

15. Sebastiani G. noninvasive assessment of liver fibrosis in chronic liver diseases: implementation in clinical practice and decision algorithms. World $\mathrm{J}$ Gastroenterol. 2009;(18):2190-203.

16. Shaikh S, Memon MS, .Ghani H, Jffery M, Shaikh K. Validation of three noninvasive markers in assessing the severity of liver fibrosis in chronic hepatitis c. $J$ Coll Physicians. Surg Pak 2009;19(8):478-82.

17. Wai CT, Greenson JK, Fontana RJ, Kalbfleisch JD, Marrero JA, Conje everam HS et al. A simple noninvasive index can predict both significant fibrosis and cirrhosis in patients with chronic hepatitis c. Hepatology.2003;38(2):518-26.

18. Wilson LE, Torbenson M,Astemborski J,Faruki $H$, Spoler C, Rai R, et al. Progression of liver fibrosis among injection drug users with chronic hepatitis $\mathbf{C}$. Hepatology.2006;43(4):788-95. 


\section{PREVIOUS RELATED STUDY}

Dilshad Muhammad, Khalid Amin, Amin Anjum, Masood Javed. CHRONIC HEPATITIS C VIRUS INFECTION; ASSOCIATION WITH TYPE 2 DIABETES MELLITUS (Original) Prof Med Jour 17(4) 557-562 Oct, Nov, Dec 2010.

Khalid Amin, Israr Kafeel, Zafar Alam, Muhammad Zakria, Masood Javaid. ROLE OF AMANTADINE IN TREATMENT OF CHRONIC HEPATITIS C (Original) Prof Med Jour 11(3) 253-260 Jul, Aug, Sep, 2004.

Muhammad Afzal, Irfan Ahmed Mughal, Naushen Afzal, Muhammad Badar Bashir, Fiyaz Ahmed Malik. CHRONIC HEPATITIS C INFECTION; CORRELATION OF GLUCOSE TOLERANCE TEST, THE LEVELS OF FASTING BLOOD SUGAR (FBS) AND HEMOGLOBIN (Original) Prof Med Jour 11(2) 222-227 Apr, May, Jun, 2004.

Shaukat Ali, Syed Khurram Shahzad, Atiq ur Rehman Slehria. CHRONIC HEPATITIS C; RESPONSE TO INTERFERON AND RIBAVIRIN COMBINATION (Original) Prof Med Jour 17(4) 563-567 Oct, Nov, Dec 2010.

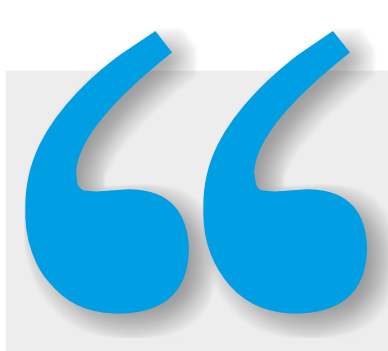

\section{"A free society is a society where it is safe to be unpopular."}

\section{Unknown}

\section{AUTHORSHIP AND CONTRIBUTION DECLARATION}

\begin{tabular}{|c|l|l|l|}
\hline Sr. \# & \multicolumn{1}{|c|}{ Author-s Full Name } & \multicolumn{1}{|c|}{ Contribution to the paper } & Author=s Signature \\
\hline 1 & Dr. Muhammad Salim Rind & 1st Author \\
2 & Dr. Muhammad lqbal Shah & 2nd Author \\
3 & Dr. Ramesh Kumar Suthar & 3rd Author \\
4 & Syed Jahangir & 4th Author
\end{tabular}

